\title{
Outcomes of patients with hematologic malignancies and COVID-19 from the Hematologic Cancer Registry of India
}

(c) The Author(s) 2021

Blood Cancer Journal (2022)12:2 ; https://doi.org/ 10.1038/s41408-021-00599-w

\section{Dear Editor,}

Several registry studies from high socio-demographic Index (SDI) countries have shown that COVID-19 illness in patients with hematological malignancies is associated with worse outcomes [1-6]. There are limited data regarding the outcomes of such patients from low SDI countries [7]. To understand the real-world outcome of COVID-19 patients with hematologic malignancies(HM) from India, the COVID-19 Hematologic Cancer Registry of India (CHCRI) started collecting data from patients of all ages with a current or history of $\mathrm{HM}$ and a laboratory-confirmed (positive RT-PCR or antigen test) COVID-19 infection from ten tertiary referral centers across India. These patients either attended the outdoor clinic or were admitted to these hospitals with COVID-19 illness. The current analysis describes the outcome of patients reported to the registry from March 21st, 2020, till March 20th, 2021. Data were submitted via individual case entry through an online CHCRI case record form maintained by Hematology Cancer Consortium(HCC). The cases from each site were not necessarily consecutive, and the denominator of cases at each site is not known. Data collection was retrospective from 21st March 2020 till 30th October 2020 and prospective from 1st November 2020 till 20th March 2021. The status of all patients was updated on May 1st, 2021. Double entries, nonhematologic diagnoses, and entries with incomplete outcome information were excluded from the analysis. The local Institutional Review Boards (IRB) approved the study at each center, and the study procedures complied with the Helsinki declaration. Any patient who received any form of therapy for the $\mathrm{HM}$ in the past 4 weeks was defined to be on active anticancer therapy. A delay in planned anticancer therapy by more than 2 weeks was considered as "interruption", while the use of lower dose chemotherapy was considered "de-escalation". COVID-19 severity was classified as mild: asymptomatic for COVID-19-related symptoms (asymptomatic positive) or $\mathrm{SpO} 2>94 \%$ on room air; moderate: $\mathrm{SpO} 2$ ranging from 90 to $94 \%$ on room air; Severe: $\mathrm{SpO} 2<90 \%$ on room air. The disease severity was analyzed using univariate and multivariable penalized logistic regression analysis. The Kaplan Meier method was used for time-to-event outcomes, and the Log-rank test was used to compare two survival curves. The variables significant at $p$-value $<0.05$ were included in the multivariable Cox regression model.

Table 1 shows the baseline study characteristics and mortality among the three COVID-19 severity categories. Among the 565 patients of COVID-19 with HM reported to the registry over 12 months, 379 (67.1)\% patients had mild COVID-19 while 71 $(12.6 \%)$ and $115(20.5 \%)$ patients had moderate and severe
COVID-19, respectively. Seventy-six percent of these patients were admitted to the hospital (Supplementary Fig. 1, 2). The mean age of the whole cohort was $41(S D \pm 19)$ years, and the male: female ratio was $2.2: 1$. In the entire cohort, the three common diagnoses in order of prevalence were Acute Lymphoblastic Leukemia (ALL), lymphoma, and Multiple Myeloma. Most of the patients $(66 \%)$, had COVID-19 within six months of being diagnosed with HM. $30 \%$ of patients were in remission for their $\mathrm{HM}$ at the time of COVID-19 diagnosis. $76 \%$ of patients were receiving active anticancer therapy when COVID-19 was diagnosed. $50.5 \%$ of patients received steroids, and $15.2 \%$ had received monoclonal antibodies in the previous 4 weeks as a part of their treatment for HM.

The diagnosis of COVID-19 had a significant impact on anticancer therapy, which was interrupted and or de-escalated in $71 \%$ of cases. The treatment of COVID-19 consisted of antiviral remdesivir in $21 \%$, steroids in $44 \%$, and prophylactic anticoagulation in $22 \%$ of patients. Less than $5 \%$ of patients received favipiravir, hydroxychloroquine (HCQ), tocilizumab, therapeutic anticoagulation, or convalescent plasma. $15.5 \%$ of patients in the whole cohort received High flow nasal cannula (HFNC) and/or ventilatory support. Among the patients with moderate/severe COVID-19, none of the COVID-19 directed drugs were associated with decreased mortality (Supplementary Table 1).

In consultation with the patient and their family members, the treating physician decided to withhold intensive chemotherapy and continue palliative therapy in $16.5 \%$ of patients. At a median follow-up of 90 days (IQR 42-180), 116 patients (20.5\%) expired, of which 75 (64.7\%) patients had severe COVID-19. Among all the patients who did not survive, $60 / 116$ (51.7\%) expired within 14 days from the COVID-19 diagnosis. Twenty-six patients $(4.6 \%)$ in the cohort had received a Hematopoietic stem cell transplant $(\mathrm{HCT})$, of which 11 patients died. $54.5 \%(6 / 11)$ of post-HCT patients who died had a severe COVID-19 illness.

On univariate analysis, age $>60$ years (HR 2.26, 1.21-4.23), diagnosis of acute myeloid leukemia (HR 3.27, 1.89-5.68), anticancer therapy interruption/ de-escalation (HR 2.43, 1.46-4.02), post-HCT status (HR 2.83, 1.51-5.28), absolute neutrophil count $(\mathrm{ANC})<0.5 \times 10^{9} / \mathrm{L}$ (HR 1.71, 1.05-2.77), plasma D-dimer $>2000 \mathrm{ng} / \mathrm{ml}$ (HR 3.06, 1.71-5.47), serum ferritin $\geq$ $500 \mathrm{ng} / \mathrm{ml}$ (HR 3.15, 1.35-7.35) were the factors associated with increased mortality. Age > 60 years (HR 2.55, 1.23-5.27), diagnosis of acute myeloid leukemia (HR 2.85, 1.58-5.13), postHCT status (HR 3.68, 1.82-7.45), and anticancer therapy interruption or de-escalation (HR 2.78, 1.65-4.68) were the significant factors for mortality on multivariable analysis (Table 2, Supplementary Fig. 3). In contrast, increasing age [20-40 years (OR 2.60 $(1.31-5.15), 40-60$ years (OR 3.44, 1.60-7.41), more than 60 years (OR 5.70, 2.43-13.35)], acute myeloid leukemia (OR 2.73, 1.45-5.12), and malignancy not being in remission (OR 1.85, 
Table 1. Clinical characteristics and outcome of patients with Hematologic Malignancy and COVID-19.

\begin{tabular}{|c|c|c|c|c|c|}
\hline \multirow[t]{2}{*}{ Variables } & \multirow{2}{*}{$\begin{array}{l}\text { All patients }{ }^{a} \\
(N=565)\end{array}$} & \multicolumn{4}{|c|}{ Overall and COVID-19 severity specific mortality ${ }^{\mathrm{a}}$ [deaths/patients(\%)] } \\
\hline & & Overall $(n=565)$ & Mild $(n=379)$ & Moderate $(n=71)$ & Severe $(n=115)$ \\
\hline \multicolumn{6}{|l|}{ Age group (years) } \\
\hline$\leq 20$ & $94(16.6)$ & 15/94 (15.6) & $9 / 81(11.1)$ & $2 / 7(28.6)$ & $4 / 6(66.7)$ \\
\hline $21-40$ & $183(32.4)$ & $32 / 183(17.5)$ & $9 / 129(6.7)$ & $2 / 20(10)$ & $21 / 34(61.7)$ \\
\hline $41-60$ & $188(33.4)$ & $38 / 188(20.2)$ & $7 / 119(5.9)$ & $3 / 26(11.5)$ & $28 / 43(65.1)$ \\
\hline$>60$ & $100(17.7)$ & $31 / 100(31)$ & $5 / 50(10)$ & $4 / 18(22.2)$ & $22 / 32(68.8)$ \\
\hline Male & $392(69.4)$ & $79 / 392(20.1)$ & $15 / 257(5.8)$ & $6 / 49(12.2)$ & $58 / 86(67.4)$ \\
\hline Female & $173(30.6)$ & $37 / 173(21.4)$ & $15 / 122(12.3)$ & $5 / 22(22.7)$ & $17 / 29(58.6)$ \\
\hline Diabetes & $81(14.3)$ & $20 / 81(24.7)$ & $2 / 44(4.6)$ & $2 / 11(18.2)$ & $16 / 26(61.5)$ \\
\hline Hypertension & $71(12.6)$ & 13/71 (18.3) & $1 / 38(2.6)$ & $1 / 9(11.1)$ & $11 / 24(45.8)$ \\
\hline \multicolumn{6}{|l|}{ Hematologic malignancy subtype } \\
\hline Hodgkin lymphoma & $27(4.8)$ & $3 / 27(11.1)$ & $1 / 20(5)$ & $1 / 4(25)$ & $1 / 3(33.3)$ \\
\hline Multiple myeloma & $93(16.5)$ & $20 / 93(21.5)$ & $3 / 53(5.7)$ & $2 / 16(12.5)$ & $15 / 24(62.5)$ \\
\hline Chronic myeloid leukemia & $23(4.1)$ & $3 / 23(13)$ & $0 / 16(0)$ & $0 / 3(0)$ & $3 / 4(75)$ \\
\hline Others $^{c}$ & $15(2.7)$ & $3 / 15(20)$ & $2 / 13(15.4)$ & $0 / 1(0)$ & $1 / 1(100)$ \\
\hline $\begin{array}{l}\text { Malignancy diagnosis to COVID-19 diagnosis interval } \\
\leq 6 \text { months }\end{array}$ & $370(65.5)$ & 70/370 (18.9) & $17 / 250(6.8)$ & $8 / 487)$ & $45 / 72(62.5)$ \\
\hline $\begin{array}{l}\text { Malignancy in remission at the time of COVID-19 } \\
\text { diagnosis }\end{array}$ & $149 / 552(27)$ & $22 / 149(14.8)$ & $7 / 112(6.3)$ & $1 / 16(6.3)$ & $14 / 21(66.7)$ \\
\hline $\begin{array}{l}\text { Systemic anticancer therapy at the time of COVID - } 19 \\
\text { diagnosis }\end{array}$ & $427 / 564(75.7)$ & $85 / 427(20)$ & $20 / 288(6.9)$ & $8 / 51(15.7)$ & $57 / 88(64.8)$ \\
\hline Malignancy therapy Interruption/de-escalation & $398 / 563(70.7)$ & $97 / 398(24.4)$ & $20 / 251(8)$ & $8 / 47(17.0)$ & $69 / 100(69)$ \\
\hline Decision forgoing ICU in favor of Palliation & $42 / 254(16.5)$ & $37 / 42(88)$ & $9 / 9(100)$ & $2 / 4(50)$ & $26 / 29(89.7)$ \\
\hline Absolute lymphocyte count $<0.5 \times 10^{9} / \mathrm{L}$ & $134 / 371(36.1)$ & $31 / 134(23.1)$ & $2 / 80(2.5)$ & $5 / 20(25)$ & $24 / 34(70.6)$ \\
\hline D-dimer $>2000 \mathrm{ng} / \mathrm{ml}$ & $55 / 235(23.4)$ & $23 / 55(41.8)$ & $2 / 24(8.3)$ & $2 / 8(25)$ & $19 / 23(82.6)$ \\
\hline C-Reactive Protein $>20 \mathrm{mg} / \mathrm{L}$ & $98 / 224(43.8)$ & 28/98(28.6) & 2/45 (4.4) & $1 / 18(5.6)$ & $25 / 35(71.4)$ \\
\hline Ferritin $\geq 500 \mathrm{ng} / \mathrm{ml}$ & $171 / 231(74)$ & $51 / 171(29.8)$ & $5 / 80(6.3)$ & $8 / 33(24.2)$ & $38 / 58(65.5)$ \\
\hline \multicolumn{6}{|l|}{ COVID-19 specific treatment } \\
\hline Steroid & $243 / 558(43.6)$ & $70 / 243(28.8)$ & $7 / 102(6.9)$ & $6 / 52(11.5)$ & $57 / 89(64)$ \\
\hline Remdesivir & $114 / 555(20.5)$ & $42 / 114(36.8)$ & $3 / 28(10.7)$ & 4/29 (13.8) & $35 / 57(61.4)$ \\
\hline Favipiravir & $17 / 555(3.1)$ & $2 / 17(11.8)$ & $1 / 12(8.3)$ & $0 / 3(0)$ & $1 / 2(50)$ \\
\hline Hydroxychloroquine & $11 / 555(2)$ & $2 / 11(18.1)$ & $0 / 5(0)$ & $0 / 2(0)$ & $2 / 4(50)$ \\
\hline Tocilizumab & $15 / 553(2.7)$ & $8 / 15(53.3)$ & $0 / 1(0)$ & $1 / 2(50)$ & $7 / 12(58.3)$ \\
\hline Prophylactic anticoagulant & $124 / 555(22.3)$ & $36 / 124(29)$ & $1 / 41(2.4)$ & $5 / 33(15.2)$ & $30 / 50(60)$ \\
\hline Therapeutic anticoagulant & $26 / 555(4.7)$ & $9 / 26(34.5)$ & $0 / 8(0)$ & $0 / 4(0)$ & 9/14 (64.3) \\
\hline Convalescent plasma & $3 / 556(0.5)$ & $2 / 3(66.7)$ & $0 / 1(0)$ & $0 / 0$ & $2 / 2(100)$ \\
\hline High flow nasal canula/ventilatory support & $86 / 556(15.5)$ & $58 / 86(67.4)$ & $0 / 0$ & $6 / 16(37.5)$ & $52 / 70(74.3)$ \\
\hline
\end{tabular}


Table 2. Univariate and multivariable cox regression analysis of factors affecting mortality ${ }^{\mathrm{a}}$.

\begin{tabular}{|c|c|c|c|c|}
\hline \multirow[t]{2}{*}{ Variables } & \multicolumn{2}{|c|}{ Univariate model for mortality } & \multicolumn{2}{|c|}{ Multivariable model for mortality } \\
\hline & HR $(95 \% \mathrm{CI})$ & $P$-Value & HR $(95 \% \mathrm{CI})$ & $P$-Value \\
\hline \multicolumn{5}{|l|}{ Age } \\
\hline$\leq 20$ & 1.00 & & 1.00 & \\
\hline $41-60$ & $1.38(0.76-2.51)$ & 0.296 & $1.50(0.75-3.00)$ & 0.255 \\
\hline$>60$ & $2.26(1.21-4.23)$ & 0.011 & $2.55(1.23-5.27)$ & 0.012 \\
\hline \multicolumn{5}{|l|}{ Hematologic malignancy related factors } \\
\hline \multicolumn{5}{|l|}{ Malignancy subtype } \\
\hline Acute lymphoblastic leukemia & 1.00 & & 1.00 & \\
\hline Acute myeloid leukemia & $3.27(1.89-5.68)$ & $<0.001$ & $2.85(1.58-5.13)$ & $<0.001$ \\
\hline Non-Hodgkin lymphoma (low grade) ${ }^{b}$ & $1.22(0.56-2.65)$ & 0.613 & $0.87(0.37-2.05)$ & 0.746 \\
\hline Others ${ }^{c}$ & $1.47(0.44-4.91)$ & 0.533 & $1.19(0.34-4.13)$ & 0.786 \\
\hline \multicolumn{5}{|c|}{ Malignancy diagnosis to COVID-19 diagnosis interval } \\
\hline$<6$ Months & 1.00 & & & \\
\hline$>6$ Months & $1.32(0.9-1.94)$ & 0.151 & - & - \\
\hline \multicolumn{5}{|l|}{ Malignancy status } \\
\hline Not in remission & $1.57(0.98-2.51)$ & 0.059 & - & - \\
\hline Remission & 1.00 & & & \\
\hline \multicolumn{5}{|c|}{ Malignancy therapy interruption or de-escalation } \\
\hline Yes & $2.43(1.46-4.02)$ & 0.001 & $2.78(1.65-4.68)$ & $<0.001$ \\
\hline No & 1.00 & & & \\
\hline \multicolumn{5}{|l|}{ Systemic anticancer therapy } \\
\hline No & 1.00 & & & \\
\hline \multicolumn{5}{|l|}{ Status post stem cell transplant } \\
\hline Yes & $2.83(1.51-5.28)$ & 0.001 & $3.68(1.82-7.45)$ & $<0.001$ \\
\hline No & 1.0 & & & \\
\hline \multicolumn{5}{|c|}{ Laboratory parameters at COVID-19 diagnosis } \\
\hline \multicolumn{5}{|c|}{$D$-dimer $(n g / m L)$} \\
\hline$<1000$ & 1.00 & & & \\
\hline $1000-2000$ & $1.19(0.49-2.91)$ & 0.701 & - & - \\
\hline$>2000$ & $3.06(1.71-5.47)$ & $<0.001$ & - & - \\
\hline \multicolumn{5}{|l|}{ Ferritin $(n g / m l)$} \\
\hline$<500$ & 1.00 & & & \\
\hline$\geq 500$ & $3.15(1.35-7.35)$ & 0.008 & - & - \\
\hline \multicolumn{5}{|l|}{ Absolute neutrophil count } \\
\hline$\geq 0.5 \times 10^{9} / \mathrm{L}$ & 1.00 & & & \\
\hline$<0.5 \times 10^{9} / \mathrm{L}$ & $1.71(1.05-2.77)$ & 0.032 & - & - \\
\hline
\end{tabular}

Bold values indicate statistical significance. 
1.18-2.89) were the factors that were significantly associated with risk of developing severe COVID-19 on multivariable analysis. Recent exposure to corticosteroids or monoclonal antibodies was not related to the risk of developing severe COVID-19 in the current cohort (Supplementary Table 2).

The study presents registry-based data of patients with HM and COVID-19 from one of the worst-hit low SDI countries in the initial 12 months. Several registry-based retrospective studies are describing the short-term follow-up (up to 6 weeks) outcomes of patients with HM and COVID-19 [5, 6, 8-11]. Most of these studies are from regions with well-equipped healthcare resources and predominantly include patients with severe COVID-19 (>70\% in most studies). The mortality in hospitalized/ severe COVID-19 in the high SDI countries exceeds $30 \%$ (Supplementary Table 3). We noted a mortality rate of $20.5 \%$ in our whole cohort that had $67 \%$ patients with mild COVID-19. The mortality was $\sim 8 \%, 16 \%$, and $65 \%$ in patients with mild, moderate, and severe COVID-19, respectively. Age $>60$ years is a common factor affecting mortality in the current as well as other studies. However, concerning the risk of developing severe COVID-19, any age $>20$ years was found significant in the current study. Most studies concur that patients with Acute Myeloid Leukemia (AML), lymphoma, and Multiple Myeloma have an increased risk for COVID-19 mortality $[6,9,11,12]$. In the current study, AML was associated with an increased risk of mortality, while low-grade lymphoma including Chronic Lymphocytic Leukemia and Multiple Myeloma were only associated with an increased risk of severe COVID-19. A quarter of AML patients died within a month of COVID-19 diagnosis; the risk of death in multiple myeloma patients was uniform over the six-month follow-up period (Supplementary Table 4). In the current study, the use of steroids or monoclonal antibodies as a part of treatment for underlying malignancy was not associated with either COVID-19 severity or mortality. These results have been contradictory in lymphoma patients in different studies [12, 13]. With regards to outcomes in post-HCT patients, our study concurs with the CIBMTR and EBMT studies, which also showed an increased risk of COVID-19 mortality post-HCT [14, 15].

Small patient numbers and prescriber biases in treatment and access may have contributed to the lack of association with COVID-19 specific therapies like antivirals, steroids, or anticoagulation and mortality. Two Spanish studies also have shown a contrary association of steroids with mortality [10, 11]. Frequently, patients with $\mathrm{HM}$ are already on steroids for their malignancy at the time of COVID-19 diagnosis; this may also confound outcomes.

The significant limitations of this study are referral bias, differential access to healthcare, heterogeneous treatment policies with the data source limited to tertiary referral centers. The true denominator of cases from a given site was not known. Though treatment interruption or de-escalation was not prospectively captured in a fraction of patients, the study does indicate the need to keep the interruptions/de-escalations to a minimum to avoid the increased risk of mortality, specifically in those patients not in remission. The strengths of the study include a large data set from one of the severely COVID-19 hit, low SDI regions [16]. Additionally, this study comprises broad hematological malignancies including leukemia, lymphoma, and myeloma, with a relatively longer followup post-COVID-19. As the study included patients from India up to March 20,2021, most of the patients were unvaccinated, and therefore, the impact of vaccination in this population was not analysed. In conclusion, the study highlights the high mortality due to COVID-19 in patients with HM especially AML and post$\mathrm{HCT}$ patients. More than one billion population in India is already vaccinated and it will be interesting to know the impact of the COVID-19 vaccine on the outcome of cancer patients in the future.
Arihant Jain ${ }^{1,12}$, Lingaraj Nayak ${ }^{2,12}$, Uday Prakash Kulkarni (iD ${ }^{3}$, Nikita Mehra ${ }^{4}$, Uday Yanamandra ${ }^{5}$, Smita Kayal ${ }^{6}$, Sharat Damodar ${ }^{7}$, Joseph M. John ${ }^{8}$, Prashant Mehta ${ }^{9}$, Suvir Singh (D) $^{10}{ }^{\prime}$, Pritesh Munot ${ }^{2}$, Sushil Selvarajan (iD ${ }^{3}$, Venkatraman Radhakrishnan ${ }^{4}$, Deepesh Lad (D) ${ }^{1}$, Rajan Kapoor ${ }^{{ }^{\prime}}$, Biswajit Dubashi ${ }^{6}$, Ram S. Bharath iD ${ }^{7}$, Hasmukh Jain ${ }^{2}$, P. K. Jayachandran ${ }^{3}$, Jeyaseelan Lakshmanan ${ }^{11}$, Thenmozhi Mani ${ }^{1{ }^{1}}$, Jayashree Thorat ${ }^{2}$, Satyaranjan Das ${ }^{5}$, Omprakash Karunamurthy ${ }^{11}{ }^{\prime}$ Biju George ${ }^{3}$, Manju Sengar ${ }^{2}$ and Pankaj Malhotra (iD ${ }^{1 \text { 的 }}$ ${ }^{1}$ Department of Clinical Hematology and Medical Oncology, Postgraduate Institute of Medical Education and Research, Chandigarh, India. ${ }^{2}$ Adult Haematolymphoid Disease Management Group, Tata Memorial Centre, Mumbai, India. ${ }^{3}$ Department of Haematology, Christian Medical College, Vellore, India. ${ }^{4}$ Department of Medical Oncology, Cancer Institute (WIA), Chennai, India. ${ }^{5}$ Army Hospital (Research \& Referral), New Delhi, India. ${ }^{6}$ Department of Medical Oncology, Jawaharlal Institute of Postgraduate Medical Education \& Research, Puducherry, India. ${ }^{7}$ Department of Haematology, Mazumdar Shaw Medical Centre, Narayana Health City, Bangalore, India. ${ }^{8}$ Department of Clinical HaematologyOncology and Bone Marrow(Stem Cell) Transplantation, Christian Medical College, Ludhiana, India. ${ }^{9}$ Department of Medical Oncology, Asian Institute, Faridabad, India. ${ }^{10}$ Department of Clinical Haematology, Dayanand Medical College, Ludhiana, India.

${ }^{11}$ Department of Biostatistics, Christian Medical College, Vellore, India. ${ }^{12}$ These authors contributed equally: Arihant Jain, Lingaraj Nayak. ${ }^{\bowtie}$ email: malhotrapankaj@hotmail.com

\section{REFERENCES}

1. https://www.ashresearchcollaborative.org/s/covid-19-registry [accessed 21 June 2021].

2. https://www.cibmtr.org/COVID19/pages/default.aspx [accessed 21 June 2021].

3. Lee AJX, Purshouse K. COVID-19 and cancer registries: learning from the first peak of the SARS-CoV-2 pandemic. Br J Cancer. 2021;124:1777-84.

4. Lee LYW, Cazier JB, Starkey T, Briggs SEW, Arnold R, Bisht V, et al. COVID-19 prevalence and mortality in patients with cancer and the effect of primary tumour subtype and patient demographics: a prospective cohort study. Lancet Oncol. 2020;21:1309-16.

5. Vijenthira A, Gong IY, Fox TA, Booth S, Cook G, Fattizzo B, et al. Outcomes of patients with hematologic malignancies and COVID-19: a systematic review and meta-analysis of 3377 patients. Blood. 2020;136:2881-92.

6. Pagano L, Salmanton-García J, Marchesi F, Busca A, Corradini P, Hoenigl M, et al. COVID-19 infection in adult patients with hematological malignancies: a European Hematology Association Survey (EPICOVIDEHA). J Hematol Oncol. 2021;14:168.

7. Ranganathan $P$, Sengar $M$, Chinnaswamy G, Agrawal $G$, Arumugham $R$, Bhatt $R$, et al. Impact of COVID-19 on cancer care in India: a cohort study. Lancet Oncol. 2021;22:970-6.

8. Wood WA, Neuberg DS, Thompson JC, Tallman MS, Sekeres MA, Sehn LH, et al. Outcomes of patients with hematologic malignancies and COVID-19: a report from the ASH Research Collaborative Data Hub. Blood Adv. 2020;4:5966-75.

9. Passamonti F, Cattaneo C, Arcaini L, Bruna R, Cavo M, Merli F, et al. Clinical characteristics and risk factors associated with COVID-19 severity in patients with haematological malignancies in Italy: a retrospective, multicentre, cohort study. Lancet Haematol. 2020;7:e737-e45.

10. Piñana JL, Martino R, García-García I, Parody R, Morales MD, Benzo G, et al. Risk factors and outcome of COVID-19 in patients with hematological malignancies. Exp Hematol Oncol. 2020;9:21.

11. García-Suárez J, de la Cruz J, Cedillo Á, Llamas P, Duarte R, Jiménez-Yuste V, et al. Impact of hematologic malignancy and type of cancer therapy on COVID-19 severity and mortality: lessons from a large population-based registry study. J Hematol Oncol. 2020;13:133.

12. Regalado-Artamendi I, Jiménez-Ubieto $A$, Hernández-Rivas JÁ, Navarro $B$, Núñez L, Alaez C, et al. Risk factors and mortality of COVID-19 in patients with lymphoma: a multicenter study. HemaSphere. 2021;5:e538.

13. Duléry R, Lamure S, Delord M, Di Blasi R, Chauchet A, Hueso T, et al. Prolonged inhospital stay and higher mortality after Covid-19 among patients with nonHodgkin lymphoma treated with B-cell depleting immunotherapy. Am J Hematol. 2021;96:934-44.

14. Sharma A, Bhatt NS, St Martin A, Abid MB, Bloomquist J, Chemaly RF, et al. Clinical characteristics and outcomes of COVID-19 in haematopoietic stem-cell transplantation recipients: an observational cohort study. Lancet Haematol. 2021;8:e185-e93. 
15. Ljungman $P$, de la Camara R, Mikulska M, Tridello G, Aguado B, Zahrani MA, et al. COVID-19 and stem cell transplantation; results from an EBMT and GETH multicenter prospective survey. Leukemia. 2021;35:2885-94.

16. https://time.com/5954416/india-covid-second-wave/ [accessed 21 June 2021].

\section{ACKNOWLEDGEMENTS}

CDMC for managing study protocol and procedures across many institutional review boards; National Cancer Grid for funding the Hematology Cancer Consortium; Sharon Lionel, Parathan Karunakaran, Ashish Bhalla, Alka Khadwal, Gaurav Prakash, Suman Pramanik, Kundan Mishra, Sanjeevan Sharma, Rajiv Kumar, Harshit Khurana, Sindhu Sharma Dahagama, Prasanth Ganesan, Shilpa Prabhu, Ketan Modak, Poojitha Byreddy, Pavitra DS,Jagdeep Singh, Davinder Paul, Kunal Jain for contributing patients.

\section{AUTHOR CONTRIBUTIONS}

A.J. and D.L. contributed to the literature search, study design, data interpretation, and writing of the report. A.J., P.M., L.N., U.K., N.M., U.Y., S.K., S.D., J.J., P.M., S.S. drafted the initial plan of the study. A.J., D.L., T.M., O.P. verified the raw data. T.M. and J.L. did the statistical plan, analysis and interpreted the data. All authors recruited participants, collected, interpreted data, wrote and revised the manuscript. A.J. and L.N. contributed equally to this manuscript. All authors had full access to the full data in the study and accepted the responsibility to submit for publication.

\section{COMPETING INTERESTS}

The authors declare no competing interests.

\section{ADDITIONAL INFORMATION}

Supplementary information The online version contains supplementary material available at https://doi.org/10.1038/s41408-021-00599-w.

Correspondence and requests for materials should be addressed to Pankaj Malhotra.

Reprints and permission information is available at http://www.nature.com/ reprints

Publisher's note Springer Nature remains neutral with regard to jurisdictional claims in published maps and institutional affiliations.

(1)

Open Access This article is licensed under a Creative Commons Attribution 4.0 International License, which permits use, sharing, adaptation, distribution and reproduction in any medium or format, as long as you give appropriate credit to the original author(s) and the source, provide a link to the Creative Commons license, and indicate if changes were made. The images or other third party material in this article are included in the article's Creative Commons license, unless indicated otherwise in a credit line to the material. If material is not included in the article's Creative Commons license and your intended use is not permitted by statutory regulation or exceeds the permitted use, you will need to obtain permission directly from the copyright holder. To view a copy of this license, visit http://creativecommons. org/licenses/by/4.0/.

(c) The Author(s) 2021 